\title{
WEAK COMPACTNESS IN NORMED LINEAR SPACES
}

D. G. TACON

(Received 1 May 1970)

Communicated by B. Mond

The notion of weak compactness plays an important role in normed linear space theory. However in many instances norm completeness of the space is an important assumption for obtaining relevant theorems. This note generalises the concept of weak compactness for subsets of normed linear spaces and obtains generalisations of known theorems including that of Eberlein [2]. We use the methods and techniques of "non-standard analysis" in the proofs; several useful non-standard results for normed linear spaces are given.

The author thanks Dr. R. W. Cross under whose supervision this work was done towards a doctoral thesis at the Australian National University.

\section{Preliminaries and summary}

The reader is assumed to be familiar with the type-theoretic approach to non-standard analysis developed by Abraham Robinson in his book [7]: a brief informal description of non-standard analysis may be found in Robinson's paper [8, section 2]. Unless otherwise stated we assume $X$ to be a real infinite dimensional normed linear space. As in Robinson [7, p. 177] we assume that $X$ together with the real number system $R$ is embedded in some full structure $M$, and we develop the non-standard theory of $X$ in an enlargement ${ }^{*} M$ of $M . K$ denotes the set of all stratified statements which are admissible and hold in $M$. We denote non-standard entities in ${ }^{*} M$ by using, as in, e.g., $x \in{ }^{*} X$ and $F \subset{ }^{*} X$. Furthermore when there is no confusion we omit the asterisk from standard entities in ${ }^{*} M$; e.g., we write

$$
\text { " }|f(x-x)|<\varepsilon, f \in S^{\prime \prime},
$$

when we should properly write

$$
\text { ،* }\left|{ }^{*} f\left({ }^{*} x-x\right)\right| *<* \varepsilon,{ }^{*} f^{*} \in{ }^{*} S^{\prime},
$$

(see [7, p. 43]). Finally we write $\boldsymbol{a} \simeq \boldsymbol{b}$ whenever $\boldsymbol{a}, \boldsymbol{b} \in *^{*} R$ and $\boldsymbol{a}-\boldsymbol{b}$ is infinitesimal [7, p. 43]. Our standard terminology and notation is generally that of 
[1]. However we use $X^{\prime}, X^{\prime \prime}$, and $X^{\prime \prime}$ to denote the first, second and third conjugate spaces of $X$ respectively.

Let $A$ be a subset of $X$. Corresponding to a map $\phi$ from the convex hull of $A, \operatorname{co}(A)$, into the set of subsets of the unit sphere $S^{\prime}$ of $X^{\prime}$ and to a given $\varepsilon>0$ let

$$
U_{x}(\phi, \varepsilon)=x+\{y:|f(y)|<\varepsilon, \quad f \in \phi(x)\} .
$$

The system of weakly open sets $\left\{U_{x}(\phi, \varepsilon): x \in \operatorname{co}(A)\right\}$ forms a covering of $A$, which we call the $(\phi, \varepsilon)$-covering of $A$.

Definition. Let $A$ be a subset of a normed linear space $X$. We say $A$ is nearly weakly compact if each $(\phi, \varepsilon)$-covering of $A$ has a finite subcover; i.e., there exist points $x_{1}, \cdots, x_{n}$ in $\operatorname{co}(A)$ such that

$$
A \subset x_{1}(\phi, \varepsilon) \cup U_{x_{2}}(\phi, \varepsilon) \cup \cdots \cup U_{x_{n}}(\phi, \varepsilon) .
$$

We prove the following result in section 2.

Let $A$ be a subset of a normed linear space $X$. Then the following statements are equivalent:

(i) $\hat{A}$ is weakly sequentially compact in $X^{\prime \prime}$,

(ii) the weak closure of $A$ is nearly weakly compact.

As a corollary we obtain a non-standard proof of Eberlein's theorem [2]. Recent (standard) proofs have been obtained by Pełczyński [6] and Whitley [10]; see also $[1$, p. 466$]$.

In section 3 we consider the case when the unit ball is nearly weakly compact and derive necessary and sufficient conditions that $\hat{X}$ be dense in $X^{\prime \prime}$.

\section{The general case}

Robinson [7, p. 90 and p. 93] introduces the concepts of monad and nearstandardness for points in the enlargement of a topological space. If $X$ is a normed linear space it is an easy consequence of the definition of the weak topology that a point $x \in{ }^{*} X$ is near-standard in the weak topology if and only if there is an $x \in X$ such that $f(x) \simeq f(x)$ for all $f \in X^{\prime}$. We generalise this property of points of ${ }^{*} X$ in the following way.

Definition. Let $X$ be a normed space. We say the point $x \in{ }^{*} X$ is nearly weak near-standard if for each $\varepsilon>0$ there is an $x \in X$ such that for all $f \in S^{\prime}$ $|f(\boldsymbol{x}-x)|<\varepsilon$.

We note an easy result.

Lemma 1. (Non-standard) Let $A$ be a subset of $X$. A point $x \in *^{*} A$ is nearly weak near-standard if and only if there is a sequence $\left\{x_{n}\right\}$ in $\operatorname{co}(A)$ such that for each $n\left|f\left(x_{n}-x\right)\right|<1 / n$ for all $f \in S^{\prime}$. 
Proof. The sufficiency of the condition is immediate.

Suppose then that $x$ is nearly weak near-standard. Then there is a sequence $\left\{y_{n}\right\}$ of $X$ such that

$$
\left|f\left(y_{n}-\boldsymbol{x}\right)\right|<\frac{1}{2 n}
$$

for all $f \in S^{\prime}$ and $n=1,2, \cdots$. But $\left\{y_{n}\right\}$ is a Cauchy sequence for

$$
\left\|y_{n}-y_{m}\right\|=\sup \left\{\left|f\left(y_{n}\right)-f\left(y_{m}\right)\right|: f \in S^{\prime}\right\}<\frac{1}{2 n}+\frac{1}{2 m}
$$

and so $\left\{\hat{y}_{n}\right\}$ is convergent to an element $y^{\prime \prime} \in X^{\prime \prime}$. Since $y^{\prime \prime}$ is near-standard in the weak topology to $\hat{x}, y^{\prime \prime}$ belongs to the weak closure of $\hat{A}[7$, p. 91], and consequently to $\overline{\operatorname{co}}(\hat{A})[1$, p. 422$]$. As

$$
\left\|\hat{y}_{n}-y^{\prime \prime}\right\|<\frac{1}{2 n} \text { for } n=1,2, \cdots,
$$

there exists a sequence $\left\{x_{n}\right\}$ in $\operatorname{co}(A)$ such that

$$
\left\|x_{n}-y_{n}\right\|<\frac{1}{2 n} \text { for } n=1,2, \cdots .
$$

But then for each $n\left|f\left(x_{n}-x\right)\right|<1 / n$ for all $f \in S^{\prime}$.

Theorem 1 generalises a well-known result of Robinson [7, p. 93].

THEOREM 1. (Non-standard). A subset $A$ of $X$ is nearly weakly compact if and only if each point $\boldsymbol{x} \in *^{*} A$ is nearly weak near-standard.

Proof. Suppose there exists an $x \in{ }^{*} A$ which is not nearly weak near-standard. Then by Lemma 1 there is a positive $\varepsilon$ such that for each $x \in \operatorname{co}(A)$, there is an $f \in S^{\prime}$ such that

$$
|f(x-x)|>\varepsilon
$$

i.e. there is a map $\phi$ such that

$$
\boldsymbol{x} \notin * U_{x}(\phi, \varepsilon)
$$

for each $x \in \operatorname{co}(A)$. But $A$ is nearly weakly compact, so that there is a finite subset $x_{1}, \cdots, x_{n}$ of $\operatorname{co}(A)$ such that

$$
A \subset U_{x_{1}}(\phi, \varepsilon) \cup U_{x_{2}}(\phi, \varepsilon) \cup \cdots \cup U_{x_{n}}(\phi, \varepsilon) .
$$

Now this equation can be formulated as a sentence of $K$, which interpreted in ${ }^{*} M$ yields

$$
{ }^{*} A \subset{ }^{*} U_{x_{1}}(\phi, \varepsilon) \cup{ }^{*} U_{x_{2}}(\phi, \varepsilon) \cup \cdots \cup{ }^{*} U_{x_{n}}(\phi, \varepsilon) .
$$

But $x \in{ }^{*} A$, whilst by assumption $x$ does not belong to any of the set $s$ on the right hand side. Hence we have a contradiction which shows the condition is necessary. 
On the other hand suppose $A$ is not nearly weakly compact. Then there exist a map $\phi$ and $\varepsilon>0$ such that the $(\phi, \varepsilon)$-covering of $A, \psi$, has no finite subcover of $A$. We define a binary relation $R(U, y)$ in $M$ such that $R(U, y)$ holds if and only if $U \in \psi$ and $y \in A$ but $y \notin U$. By assumption $R(U, y)$ is concurrent, so that by definition of ${ }^{*} M$ there is a point $\boldsymbol{x}$ in ${ }^{*} A$ such that

$$
x \notin U_{x}(\phi, \varepsilon)
$$

for all $x \in \operatorname{co}(A)$. But then $x$ is not nearly weak near-standard by Lemma 1 .

We remark that an analysis of this proof shows that when defining nearly weakly compact sets it suffices to consider only those $(\phi, \varepsilon)$-coverings where $\phi$ maps into singletons of $S^{\prime}$.

COROLlary 1. (Standard) Let $A$ be a weakly closed subset of a normed linear space $X$. If $A$ is nearly weakly compact then $A$ is weakly compact provided $\overline{\mathrm{co}}(A)$ is complete.

Proof. Suppose $A$ is nearly weakly compact, and that $x \in * A$. As $\operatorname{co}(A)$ is complete by the proof of Lemma 1 there is a point $x \in X$ such that $x$ is nearstandard to $x$ in the weak topology. Hence, as $A$ is weakly closed, we have $x \in A$ $[7$, p. 91]. But then $A$ is weakly compact $[7$, p. 94].

A point $x \in{ }^{*} X$ is finite if there is a (standard) real number $m$ such that $\|\boldsymbol{x}\| \leqq m$.

Lemma 2. (Non-standard) Let $\boldsymbol{x}$ be a finite point in $* X$. Then $\boldsymbol{x}$ is nearly weak near-standard if and only if for each integer $n$, there is a finite subset $C_{n}$ of $X$ such that for each $f \in S^{\prime}$, there is an $x \in C_{n}$ satisfying $f(x-x)<1 / n$.

Proof. The necessity of the condition follows from the definition.

Otherwise, we define an element $x^{\prime \prime}$ of $X^{\prime \prime}$ by

$$
x^{\prime \prime}(f)=\circ[f(x)] \text {, }
$$

the standard part of $f(\boldsymbol{x})$, for each $f \in X^{\prime}$. By $[7$, p. 51$] \circ[f(x)]$ exists since $|f(x)|$ is finite. If

$$
C_{\infty}=\bigcup_{n=1}^{\infty} C_{n}
$$

and $C=\operatorname{co}\left(C_{\infty}\right)$ then $x^{\prime \prime} \in D$, the norm closure of $\hat{C}$ in $X^{\prime \prime}$. Otherwise by the separation theorem $\left[1\right.$, p. 417] there is an element $x^{\prime \prime \prime} \in X^{\prime \prime \prime}$ such that $\left\|x^{\prime \prime}\right\|<1$, together with real constants $c$ and $\varepsilon>0$, satisfying

$$
x^{m}(D) \leqq c-\varepsilon<c \leqq x^{\prime \prime}\left(x^{\prime \prime}\right) .
$$

Let $n$ be an integer greater than $1 / \varepsilon$ and consider $C_{n}=\left\{y_{1}, \cdots, y_{m}\right\}$. By Helly's theorem [11, p. 103] there exists an $f \in X^{\prime}$ with $\|f\|<1$ such that $\hat{f}=x^{\prime \prime}$ on $\hat{y}_{i}, i=1, \cdots, m$ and $x^{\prime \prime}$. Then 
so that

$$
f\left(y_{i}\right)+\varepsilon<x^{\prime \prime}(f) i=1, \cdots, m
$$

$$
f(x)-f\left(y_{i}\right)>1 / n i=1, \cdots, m .
$$

which is a contradiction. As $x^{\prime \prime} \in D, \boldsymbol{x}$ is nearly weak near-standard, for if $\left\|\hat{x}-x^{\prime \prime}\right\|<\varepsilon$, then

$$
|f(x)-f(x)|<\varepsilon
$$

for all $f \in S^{\prime}$.

THEOREM 2. (Standard) Let $A$ be a subset of a normed linear space. If $\hat{A}$ is weakly sequentially compact, then the weak closure of $A$ is nearly weakly compact.

Proof. (Non-standard) Let $\bar{A}$ be the closure of $A$ in the weak topology. Then $\bar{A}$ is norm bounded by the uniform boundedness principle, so that all points $\boldsymbol{x} \in{ }^{*} \bar{A}$ are finite [7, p. 100]. If $\bar{A}$ is not nearly weakly compact there is a point $\boldsymbol{x} \in * \bar{A}$ which is not nearly weak near-standard. This means by Lemma 2 there is an $\varepsilon>0$ such that for each finite subset $C$ of $X$ there is an $f \in S^{\prime}$ such that for all $x \in C, f(x-x)>2 \varepsilon$.

We construct three sequences $\left\{x_{n}\right\} \subset \bar{A},\left\{y_{n}\right\} \subset A$ and $\left\{f_{n}\right\} \subset S^{\prime}$ in the following manner. We choose $x_{0}=y_{0}$ arbitrarily in $A$, then $f_{0} \in S^{\prime}$ such that

$$
f_{0}\left(y_{0}-\boldsymbol{x}\right)>2 \varepsilon \text {. }
$$

Now the statement

$$
\exists x\left(x \in \bar{A} \wedge\left(f_{0}\left(y_{0}-x\right)>2 \varepsilon\right)\right)
$$

holds in ${ }^{*} M$ (for $\boldsymbol{x}$ satisfies it), and so is true in $M$. Hence there is an $x_{1} \in A$ such that

$$
f_{0}\left(y_{0}-x_{1}\right)>2 \varepsilon \text {. }
$$

As $x_{1} \in A$ there exists $y_{1} \in A$ such that $\left|f_{0}\left(x_{1}-y_{1}\right)\right|<\varepsilon$. Suppose now we have chosen $y_{k}, x_{k}$ for $k=0,1, \cdots, n-1$ and $f_{j}$ for $j=0,1, \cdots, n-2$ satisfying

$$
\begin{array}{ll}
f_{j}\left(y_{i}-\boldsymbol{x}\right)>2 \varepsilon & i=0,1, \cdots, j \\
f_{j}\left(y_{i}-x_{k}\right)>2 \varepsilon & 0 \leqq i \leqq j<k \leqq n-1 \\
\left|f_{j}\left(y_{i}-x_{i}\right)\right|<\varepsilon & j=0,1, \cdots, i-1 .
\end{array}
$$

Then we choose $f_{n-1} \in S^{\prime}$ such that $f_{n-1}\left(y_{j}-\boldsymbol{x}\right)>2 \varepsilon, j=0,1, \cdots, n-1$. The abbreviated statement

$$
\exists x\left((x \in \bar{A}) \wedge\left(f_{i}\left(y_{j}-x\right)>2 \varepsilon, 0 \leqq j \leqq i<n\right)\right)
$$

is true in $* M$ (for $x$ satisfies it) and so is true in $M$. This means we can choose $x_{n} \in \bar{A}$ such that 
Lemma 3. (Non-standard) Let $X$ be a linear space. Then there exists a "finite dimensional" subspace $F$ of ${ }^{*} X$ such that $X \subset F$; i.e., $\left\{{ }^{*} x: x \in X\right\} \subset F$.

Proof. If $X$ is finite dimensional we need only take ${ }^{*} X$ as $F$ to see that the theorem holds.

Otherwise we construct a binary relation $R(x, F)$ in $M$ which holds if and only if $x$ is an element of $X, F$ is a finite dimensional subspace of $X$, and $x$ belongs to $F . R(x, F)$ is then a concurrent relation and so there exists a "finite dimensional" subspace $F$ of ${ }^{*} X$ such that $\{* x: x \in X\} \subset F$.

When $X$ is a normed linear space Lemma 3 enables us to obtain a nonstandard version of Helly's theorem [11, p. 103].

Lemma 4. (Non-standard) Let $X$ be a semi-normed linear space and let $x^{\prime \prime} \in X^{\prime \prime}$. Then there exists $x \in{ }^{*} X$ such that $x^{\prime \prime}(f)=f(x)$ for all $f \in X^{*}$ and such that $\left\|x^{\prime \prime}\right\| \simeq\|x\|$.

Proof. Let us rewrite Helly's theorem in $K$, and interpret its statement in the enlargement $* M$ for a suitable finite dimensional space and for a suitable positive constant. We have

$$
\begin{aligned}
& \forall F\left(F \text { is a finite dimensional subspace of } X^{\prime}\right) \wedge \\
& \forall \varepsilon(\varepsilon>0) \exists x\left(\left((x \in X) \wedge\left(\|x\|<\left\|x^{\prime \prime}\right\|+\varepsilon\right)\right) \wedge\right. \\
& \left.\left(\forall f(f \in F)\left(x^{\prime \prime}(f)=f(x)\right)\right)\right) .
\end{aligned}
$$

In ${ }^{*} M$ we may choose for $\varepsilon$ a positive infinitesimal and for $F$ a "finite dimensional" subspace $\boldsymbol{F}$ of ${ }^{*}\left(X^{\prime}\right)$ such that $X^{\prime} \subset \boldsymbol{F}$ in the sense of Lemma 3. $\boldsymbol{f} \in \boldsymbol{F}$.

Then there is an $\boldsymbol{x} \in{ }^{*} X$ such that $\left\|x^{\prime \prime}\right\| \simeq\|\boldsymbol{x}\|$ satisfying $x^{\prime \prime}(f)=\hat{f}(x)$ for all

The lemma now follows as $X^{\prime} \subset \boldsymbol{F}$.

With this representation of element of the second conjugate $X^{\prime \prime}$ we now establish the following result.

Theorem 3. (Standard) Let $X$ be a normed linear space. Then $\hat{X}$ is dense in $X^{\prime \prime}$ if and only if the unit ball $B$ of $X$ is nearly weakly compact.

Proof. (Non-standard) Suppose $\hat{X}$ is dense in $X^{\prime \prime}$, and suppose $x \in{ }^{*} B$. As in Lemma 2 we define an element of $X^{\prime \prime}$ by

$$
x^{\prime \prime}(f)=\circ[f(x)] \text {. }
$$

For a given $\varepsilon>0$ there is an $x \in B$ such that $\left\|x^{\prime \prime}-\hat{x}\right\|<\varepsilon$. But then

$$
\left|x^{\prime \prime}(f)-f(x)\right|<\varepsilon \quad \text { for all } f \in S^{\prime}
$$

so that $|f(\boldsymbol{x})-f(x)|<\varepsilon$ for $f \in S^{\prime}$. Hence $\boldsymbol{x}$ is nearly weak near-standard and so by Theorem $1, B$ is nearly weakly compact. 


$$
f_{i}\left(y_{j}-x_{n}\right)>2 \varepsilon, 0 \leqq j \leqq i<n,
$$

and in turn $y_{n} \in A$ such that

$$
\mid f_{k}\left(x_{n}-y_{n}\right)<\varepsilon, k=0,1, \cdots, n-1 \text {. }
$$

Therefore we can choose sequences $\left\{y_{n}\right\}$ in $A,\left\{f_{n}\right\}$ in $S^{\prime}$ satisfying

$$
f_{k}\left(y_{i}-y_{n}\right)>\varepsilon, \quad 0 \leqq i \leqq k<n .
$$

As $\hat{A}$ is weakly sequentially compact, $\left\{\hat{y}_{n}\right\}$ has a subsequence which tends towards an element $x^{\prime \prime}$ of $X^{\prime \prime}$ in the weak topology. Without loss of generality we may assume $\hat{y}_{n} \rightarrow x^{\prime \prime}$ in the weak topology. Then

$$
\hat{y}_{i}\left(f_{n}\right)-x^{\prime \prime}\left(f_{n}\right)>\varepsilon \quad \text { for } n \geqq i \geqq 0 .
$$

The Banach-Alaoglu theorem ensures that the sequence $\left\{\hat{f}_{n}\right\}$ has a limit point in the weak* topology, say $x^{m}$. But then

$$
x^{\prime \prime}\left(\hat{y}_{n}\right)-x^{\prime \prime \prime}\left(x^{\prime \prime}\right)>\varepsilon, \quad n=1,2, \cdots,
$$

contradicting the assumption $\hat{y}_{n} \rightarrow x^{\prime \prime}$ in the weak topology. The theorem is now proven.

The following corollary is Eberlein's theorem in a normed linear space context (see, e.g., [3, p. 551]).

COROLlARY 2. If $A$ is a weakly sequentially compact subset of a normed linear space, then the weak closure of $A$ is weakly compact provided $\overrightarrow{c o}(A)$ is complete.

Proof. The result follows by Theorem 2 and Corollary 1.

Theorem 2 has established the implication (i) implies (ii) in the result stated in section 1. The converse will follow by Šmulian's theorem [1, p. 430] if it is shown that $\hat{A}$ is weakly countably compact. By $[7, \mathrm{p} .96]$ this is the case if each $\boldsymbol{x} \in{ }^{*} \hat{A}$ is near-standard in the weak topology. This is an immediate consequence of the fact that $\boldsymbol{x}$ is nearly weak near-standard.

\section{The case of the unit ball}

Our main aim in this section is to give a non-standard proof of Theorem 3. We can do this quickly once we note a non-standard version of Helly's theorem (Lemma 4).

However we first give a simple but useful embedding result. Staples [9] has produced a similar result for Boolean algebras and has announced our result in the same paper. 
Conversely suppose $x^{\prime \prime} \in B^{\prime \prime}$. By Lemma 4 there exists an $x \in{ }^{*} B$ such that

$$
x^{* *}(f)=\circ[f(\boldsymbol{x})]
$$

for all $f \in X^{\prime}$. But $\boldsymbol{x}$ is nearly weak near-standard so that for a given $\varepsilon>0$ there exists $x \in X$ such that

$$
|f(x)-f(x)|<\varepsilon \text { for all } f \in S^{\prime} .
$$

But then $\left|x^{\prime \prime}(f)-f(x)\right| \leqq \varepsilon$ for all $f \in S^{\prime}$, so that $\left\|x^{\prime \prime}-\hat{x}\right\| \leqq \varepsilon$. Hence $\hat{X}$ is dense in $X^{\prime \prime}$.

We point out that if the unit ball of $X$ is weakly sequentially compact then $X$ is reflexive. This result was noted by Gustafson [5, p. 561], and is also a consequence of a more general result of Fan and Glicksberg [4, p. 951].

Finally we note the following result.

THEOREM 4. $\hat{X}$ is dense in $X^{\prime \prime}$ if and only if for each $x^{\prime \prime} \in X^{\prime \prime}$ and for each integer $n$ there is a finite subset $C_{n}$ of $X$ such that for each $f \in S^{\prime}$ there is an $x \in C_{n}$ satisfying $f(x)-x^{\prime \prime}(f)<1 / n$.

Proof. (Non-standard) The result follows in a manner similar to the proof of Theorem 3 when we use the characterisation of nearly weak near-standard given by Lemma 2.

\section{References}

[1] N. Dunford and J. T. Schwartz, Linear Operators, Part 1 (Interscience, New York, 1958).

[2] W. F. Eberlein, 'Weak compactness in Banach spaces', I, Proc. Nat. Acad. Sci. USA 33 (1947), 51-53.

[3] R. E. Edwards, Functional Analysis: Theory and Applications (Holt, Rinehart and Winston, Inc., New York, 1965).

[4] Ky Fan and Irving Glicksberg, 'Fully convex normed linear spaces', Proc. Nat. Acad. Sci. USA 41 (1955), 947-953.

[5] Karl Gustafson, 'Compact-like operators and the Eberlein theorem', Amer. Math. Monthly 75 (1968), 958-964.

[6] A. Pelczynski, 'A proof of the Eberlein-Šmulian theorem by an application of basic sequences', Bull. Polon. Sci. Ser. Math. Astr. et Phys. 12 (1964), 543-548.

[7] A. Robinson, Non-standard Analysis (Studies in Logic and the Foundations of Mathematics, North-Holland, Amsterdam, 1966).

[8] Abraham Robinson, 'Non-standard arithmetic', Bull. Amer. Math. Soc. 73 (1967), 818-843.

[9] John Staples, 'A non-standard representation of Boolean algebras', Bull. London Math. Soc. 1 (1969), 315-320.

[10] Robert Whitley, 'An elementary proof of the Eberlein-Šmulian theorem', Math. Annalen 172 (1967), 116-118.

[11] A. Wilansky, Functional Analysis (Blaisdell, 1964).

Australian National University

Canberra, ACT 